PROCEEDINGS OF THE

AMERICAN MATHEMATICAL SOCIETY

Volume 29, Number 1, June 1971

\title{
TAME ARCS ON WILD CELLS
}

\author{
CHARLES L. SEEBECK III ${ }^{1}$
}

\begin{abstract}
We prove here that, for $n \geqq 5$, every cell in $E^{n}$ contains a tame arc and that, for product cells $B^{m-k} \times I^{k} \subset E^{n-k} \times E^{k}$ $=E^{n}$, every $k$-dimensional polyhedron $P \subset B^{m-k} \times I^{k}$ is tame in $E^{n}$.
\end{abstract}

1. Introduction. Bing showed in [1] that every 2-cell in 3-dimensional Euclidean space contains a tame arc and in [2] that there is a 2 -sphere that is wild but for which all subarcs are tame. We obtain here analogous results in higher dimensions $(\geqq 5)$. First we show that for $n \geqq 5$, any subarc of any $k$-cell in $E^{n}$ can be approximated by subarcs tame in $E^{n}$. Then we show that if $C$ is any $(m-k)$-cell in $E^{n-k}, I^{k} \subset E^{k}$ is the $k$-fold product of the unit interval $I, m \leqq n-2$, and $n \geqq 5$, then every sub $k$-cell of $C \times I^{k} C E^{n-k} \times E^{k}$ is tame in $E^{n}$. Since there are cells in this class of factored cells that are wild at every point [10] we have a generalization of Bing's example [2] to higher dimensions.

2. The approximation theorems. First we give a few definitions. Let $X \subset M$ be closed subsets of $E^{n}$. Let $d$ denote the usual metric on $E^{n}$. A homeomorphism $h$ of $M$ is an $\epsilon$-push of $(M, X)$ if there is an isotopy $h_{t}$ of $M$ such that $h_{0}=$ Identity, $h_{1}=h, d\left(h_{t}(x), x\right)<\epsilon$ for each $t \in I$ and each $x \in M$, and $h_{t}$ is the identity outside the $\epsilon$-neighborhood of $X$ in $M$ for each $t$. If $P$ is a polyhedron and $h: P \rightarrow E^{n}$ is an embedding we say that $h$ is tame if there is a homeomorphism $H$ of $E^{n}$ such that $H \cdot h$ is piecewise linear (PL).

Lemma 1. Suppose $X$ is a compact subset of $E^{n}$, Int $X=\varnothing, X$ does not locally separate $E^{n}, G$ is a compact 1-dimensional subpolyhedron of $E^{n}, n \geqq 4$, and $\epsilon>0$. Then there is an $\epsilon$-push $h$ of $\left(E^{n}, G \cap X\right)$ such that $h(G) \cap X=\varnothing$.

Proof. The proof is an immediate consequence of general position and Corollary 5.6 of [3].

Lemma 2. Suppose $X$ is a compact subset of $E^{n}$, Int $X=\varnothing, X$ does not locally separate $E^{n}, P$ is a 2-dimensional subpolyhedron of $E^{n}, n \geqq 4$,

Received by the editors June 21, 1970.

AMS 1969 subject classifications. Primary 5478, 5570, 5705; Secondary 5701, $5720,5760$.

Key words and phrases. Tame embedding, $\epsilon$ push, 1-ULC, locally separates $E^{n}$, wild cell.

${ }^{1}$ Supported by NSF Grant GP19462.

Copyright (c) 1971, American Mathematical Society 
and $\epsilon>0$. Then there is an $\epsilon$-push $h$ of $\left(E^{n}, P \cap X\right)$ such that $h(P) \cap X$ is totally disconnected.

Proof. Let $K$ be a triangulation of $P$ and $\left\{K_{i} \mid i=1,2, \cdots\right\}$ the sequence of $i$ th derived barycentric subdivisions of $K$. We shall use Lemma 1 to construct an $\epsilon$-push $h$ of $\left(E^{n}, P \cap X\right)$ such that $h\left(U\left|k_{i}^{1}\right|\right)$ $\cap X=\varnothing$. Clearly $h$ then satisfies the conclusion of Lemma 2 .

Let $\epsilon_{1}=\epsilon / 2$ and apply Lemma 1 with $(X, G, \epsilon)$ replaced by $(X$, $\left.\left|K_{1}^{1}\right|, \epsilon_{1}\right)$, obtaining an $\epsilon_{1}$-push $h_{1}$ of $\left(E^{n},\left|K_{1}^{1}\right| \cap X\right)$ such that $h_{1}\left(\left|K_{1}^{1}\right|\right)$ $\cap X=\varnothing$. Let $\delta_{1}=d\left(h_{1}\left(\left|K_{1}^{1}\right|\right), X\right)$ and $\eta_{\mathrm{i}}$ be some positive number chosen depending on $h_{1}$. (See [8] or Theorem 3.4 of [7].) Set $\epsilon_{2}$ $=\min \left\{\epsilon_{1} / 2, \delta_{1} / 2, \eta_{1}\right\}$. As before we obtain an $\epsilon_{2}$-push $h_{2}^{\prime}$ of $\left(E^{n}\right.$, $\left.h_{1}\left|K_{2}^{1}\right| \cap X\right)$ such that $h_{2}^{\prime} \cdot h_{1}\left(\left|K_{2}^{1}\right|\right) \cap X=\varnothing$. Set $h_{2}=h_{2}^{\prime} \cdot h_{1}$. Continuing in this way we obtain a sequence $\left\{h_{i}\right\}$ of homeomorphisms of $E^{n}$. Since $\epsilon_{i+1}<\epsilon_{i} / 2, \lim _{i \rightarrow \infty} h_{i}=h$ is an $\epsilon$-map of $E^{n}$ supported on a compact set. Because $\epsilon_{i+j} \leqq \delta_{i} / 2^{j}, h\left(U\left|K_{i}^{1}\right|\right) \cap X=\varnothing$ and because the $\eta_{i}$ are chosen sufficiently small depending on the $h_{i}, h$ is a homeomorphism. Thus $h$ is the required $\epsilon$-push of $\left(E^{n}, X\right)$.

TheOREM 3. Let $M$ be a PL m-manifold topologically embedded in $E^{n}, G$ a 1-dimensional subpolyhedron of $M, G^{\prime}$ a subpolyhedron of $G$ that is tame in $E^{n}, n \geqq 5$, and $m \geqq 2$. Then for each $\epsilon>0$ there is an $\epsilon$-embedding $\alpha: G \rightarrow M$ such that $\alpha$ is tame in $E^{n}$ and $\alpha \mid G^{\prime}=$ inclusion: $G^{\prime} \rightarrow E^{n}$.

Proof. It is sufficient to consider the case that $M$ is a 2-cell, $G$ is an arc, and $G^{\prime}=\{$ end points of $G\}$. Using Lemma 2 we shall construct an embedding $\alpha: G \rightarrow M$ fixed on $G^{\prime}$ such that $E^{n}-\alpha(G)$ is uniformly locally 1 -connected (1-ULC). By Theorem 4.2 of [4], $\alpha$ is thus tame.

Let $K$ be a triangulation of $E^{n}$ and $K_{1}, K_{2}, \ldots$ the sequence of $i$ th derived barycentric subdivisions of $K$. It follows from general position and Lemma 2 that there is an $\epsilon / 2$-push $h_{1}$ of $\left(E^{n}, G \cap\left|K_{1}^{2}\right|\right)$ such that $h_{1}\left(\left|K_{1}^{2}\right|\right) \cap M$ is totally disconnected and $h_{1}\left(\left|K_{1}^{2}\right|\right) \cap G^{\prime}=\varnothing$. Thus there is an $\epsilon / 2$-embedding $\alpha_{1}: G \rightarrow M$ fixed on $G^{\prime}$ such that $\alpha_{1}(G)$ $\cap h_{1}\left|K_{1}^{2}\right|=\varnothing$. Now as in the proof of Lemma 2 we set $\epsilon_{1}=\epsilon / 2$, $\delta_{1}=d\left(\alpha_{1}(G), h_{1}\left(\left|K_{1}^{2}\right|\right)\right), \eta_{1}>0$ chosen depending on $\alpha_{1}, \eta_{1}^{\prime}>0$ chosen depending on $h_{1}, \epsilon_{2}=\min \left\{\epsilon_{1} / 2, \delta_{1} / 4, \eta\right\}$, and $\epsilon_{2}^{\prime}=\min \left\{\epsilon_{1} / 2, \delta_{1} / 4, \eta_{1}^{\prime}\right\}$. Then using Lemma 2 we find an $\epsilon_{2}^{\prime}$-push $h_{2}^{\prime}$ of $\left(E^{n}, h_{1}\left(\left|K_{2}^{2}\right|\right) \cap M\right)$ such that $h_{2}^{\prime} \cdot h_{1}\left(\left|K_{2}^{2}\right|\right) \cap M$ is totally disconnected. Let $h_{2}=h_{2}^{\prime} \cdot h_{1}$. We can again find an embedding $\alpha_{2}: G \rightarrow M$ such that $d\left(\alpha_{1}, \alpha_{2}\right)<\epsilon_{2}, \alpha_{2}(G)$ $\cap h_{2}\left(\left|K_{2}^{2}\right|\right)=\varnothing$, and $\alpha_{2}$ is fixed on $G^{\prime}$. Continuing in this way we construct a sequence $\alpha_{i}: G \rightarrow M$ of $\epsilon_{i}$-embeddings and a sequence $\left\{h_{i}\right\}$ of homeomorphisms of $E^{n}$. Because $\epsilon_{i+1} \leqq \epsilon_{i} / 2$, the $\alpha_{i}$ converge to an $\epsilon$-map $\alpha: G \rightarrow M$. The $\eta_{i}$ can be picked so as to guarantee that $\alpha$ is an 
$\epsilon$-embedding. Similarly the $h_{i}$ converge to an $\epsilon$-push of $\left(E^{n}, M\right)$. Because $\max \left\{\epsilon_{i+j}, \epsilon_{i+j}^{\prime}\right\} \leqq \delta_{i} / 2^{j+1}, \alpha(G) \cap h\left(\bigcup_{i=1}^{\infty}\left|K_{i}^{2}\right|\right)=\varnothing$. Thus $h^{-1}$ $\cdot \alpha(G) \cap \bigcup_{i=1}^{\infty}\left|K_{i}^{2}\right|=\varnothing$, and so $E^{n}-h^{-1} \cdot \alpha(G)$ is 1 -ULC. Therefore $h^{-1} \cdot \alpha$ and hence $\alpha$ is tame.

Corollary 3.1. Suppose $N$ is a PL n-manifold, $M$ is a PL m-manifold topologically embedded in $N, G$ is a 1-dimensional polyhedron, $G^{\prime} \subset G$ is a subpolyhedron, $\beta: G \rightarrow M$ is an embedding such that $\beta \mid G^{\prime}: G^{\prime}$ $\rightarrow N$ is tame, $n \geqq 5$, and $m \geqq 2$. Then for each $\epsilon>0$ there is an embedding $\alpha: G \rightarrow M$ such that $d(\alpha, \beta)<\epsilon, \alpha\left|G^{\prime}=\beta\right| G^{\prime}$, and $\alpha: G \rightarrow N$ is tame.

Proof. First take an infinite triangulation of $G-G^{\prime}$ and approximate $\beta$ by an embedding $\beta^{\prime}: G \rightarrow M$ such that $\beta^{\prime}\left|G^{\prime}=\beta\right| G^{\prime}$ and $\beta^{\prime}$ is locally PL on $G-G^{\prime}$. Then apply Theorem 3 to a sequence of compact subpolyhedra of $\beta^{\prime}\left(G-G^{\prime}\right)$. Thus we obtain an embedding $\alpha: G \rightarrow M$ such that $\alpha\left|G^{\prime}=\beta\right| G^{\prime}$ and $\alpha \mid G-G^{\prime}$ is locally tame in $E^{n}$. Thus $\alpha$ : $G$ $\rightarrow N$ is tame (Theorem 4.2 of $[4]$ ).

Theorem 4. Suppose $N$ is a PL $n$-manifold, $M$ is a PL m-manifold topologically embedded in $N$, every 2-complex of $M$ can be approximated by a 2-complex in $M$ that is tame in $N$, and $5 \leqq m \leqq n-2$. Then each $k$ dimensional polyhedron $P$ topologically embedded in $M, k<m$, can be approximated in $M$ by embeddings that are tame in $N$.

Proof. It follows from [5] and either [6] or [9] that an approximation of $P$ is tame if its complement is $1-\mathrm{ULC}$. Such an approximation is found by modifying the proof of Theorem 3 . Let $L$ be a triangulation of $M$ and $L_{1}, L_{2}, \cdots$ the sequence of barycentric subdivisions. Similarly let $K_{1}, K_{2}, \cdots$ be the sequence of barycentric subdivisions of a triangulation of $N$. Using techniques similar to those above it is possible to construct a homeomorphism $h$ of $N$ such that $h\left(U\left|K_{i}^{1}\right|\right)$ $\cap M=\varnothing$ and $h\left(\mathrm{U}\left|K_{i}^{2}\right|\right) \cap\left(\mathrm{U} Q_{i}\right)=\varnothing$ where $Q_{i}$ is a close approximation of $\left|L_{i}^{2}\right|$ for each $i$ that is tame in $N$. Now for each $i$ we can find an arc $A_{i} \subset M$ such that $C_{i}=h\left(\left|K_{i}^{2}\right|\right) \cap M \subset A_{i}$ and $A_{i}-C_{i}$ is locally tame in $M$. Since $M-C_{i}$ is 1 -ULC, $M-A_{i}$ is 1 -ULC and hence $A_{i}$ is tame. Using $A_{i}$ we can construct, for each $i$, a homeomorphism $f_{i}$ of $M$ moving points a distance depending on $f_{i-1}$ so that $f_{i}(P) \cap C_{i}=\varnothing$. Thus as in the proof of Lemma 2 and Theorem 3 we can construct an $\epsilon$-push $f$ of $(M, P)$ such that $f(P) \cap\left(U C_{i}\right)=\varnothing$. Thus $N-f(P)$ is $1-\mathrm{ULC}$ and the required approximation has been found.

It is evident that we have actually proved the following.

Addendum to Theorem 4. Under the hypotheses of Theorem 4 it is possible to find for each $\epsilon>0$ an $\epsilon$-push $f$ of $(M, P)$ such that $f \mid P: P \rightarrow N$ is tame. 
3. Subpolyhedra of factored cells. We say that an $m$-cell $C \subset E^{n}$ factors $k$-times if for some homeomorphism $h: E^{n} \rightarrow E^{n}$ and some $(m-k)$-cell $B \subset E^{n-k}, h(C)=B \times I^{k} \subset E^{n-k} \times E^{k}$ where $I^{k}$ is the $k$-fold product of the interval $I$ naturally embedded in $E^{k}$ and $B \times I^{k} C E^{n-k}$ $\times E^{k}$ is the product embedding.

Theorem 5. Suppose $C$ is an m-cell topologically embedded in $E^{n}, C$ factors $k$-times, $n \geqq 5$, and $m \leqq n-2$. Then every embedding of any compact k-dimensional polyhedron into $C$ is tame in $E^{n}$.

Proof. Let $B$ be an $(m-k)$-cell in $E^{n-k}, P$ a finite $k$-dimensional polyhedron topologically embedded in $B \times I^{k} \subset E^{n-k} \times E^{k}, n \geqq 5$, and $1 \leqq k<m \leqq n-2$. It follows from [5] and either [6] or [9] that $P$ is tame in $E^{n}$ if $E^{n}-P$ is 1-ULC. However, $E^{n}-P$ is 1 -ULC if each 2-complex in $E^{n}$ can be homotopied off $P$ by arbitrarily small homotopies. Let $K$ be a finite 2-complex. First find a very small homotopy of $|K|$ such that for some subdivision $K^{\prime}$ each 2-cell of $K^{\prime}$ either projects on to a 0 - or 1-simplex of $E^{n-k}$ or else lies in $E^{n-k} \times t$ for some $t \in E^{k}$. Since $n-k \geqq(m-k)+2 E^{n-k}-B$ is locally 0 -connected. Thus it follows that any 0 - or 1 -simplex in $E^{n-k}$ can be homotopied off $B$ by a small homotopy. Thus any 2-cell of $K^{\prime}$ that projects onto a 0 - or 1-cell of $E^{n-k}$ can be homotopied off $B \times I^{k}$. Let $\sigma$ be a 2-cell of $K^{\prime}, t \in E^{k}$, and $\sigma \subset E^{n-k} \times t$. For $n-k \geqq 4$ it follows from Lemma 2 that there is an $\epsilon$-push $h$ of $\left(E^{n-k} \times t, \sigma\right)$ such that $h(\sigma) \cap(B \times t)$ is 0 -dimensional. For $n-k=3$ we can use the techniques of the proof of Lemma 2 to find an embedding $h: \sigma \rightarrow E^{n-k} \times t$ such that $h(\sigma) \cap(B \times t)$ is 0 dimensional and $h$ is close to the inclusion of $\sigma$ into $E^{n-k} \times t$. Let $A=h(\sigma) \cap\left(B \times I^{k}\right) . A$ is a 0 -dimensional subset of $B \times t$. Let $P$ be a $k$-dimensional polyhedron topologically embedded into $B \times I^{k}$. Let $T \subset P$ be defined as follows: $x \in T$ if there is a neighborhood $U$ of $x$ in $P$ and a point $y \in E^{n-k}$ such that $U \subset y \times I^{k}$. Then $T$ is open in $P$ and $P$ is locally tame at each point of $U$ [5]. We shall construct a map $f: B \times E^{k} \rightarrow B \times E^{k}$ such that $p_{1} \cdot f=p_{1}$ where $p_{1}=$ projection: $B \times E^{k}$ $\rightarrow B, f(A) \cap P \subset T$, and $d\left(f, \operatorname{Id} \mid B \times E^{k}\right)$ is small. For each $(x, t) \in A$ $\cap(P-T)$, let $\epsilon_{x}>0$ be chosen so that for some $t_{x} \in E^{k}$ with $d\left(t_{x}, t\right)<\epsilon_{x}$ and for all $x^{\prime} \in B$ with $d\left(x^{\prime}, x\right)<\epsilon_{x},\left(x^{\prime}, t_{x}\right) \in\left(B \times E^{k}\right)-P$. Now for some finite number of $x \in B$, the $\epsilon_{x}$-neighborhoods of the $x$ 's cover $p_{1}(A \cap(P-T))$. Since $A$ is totally disconnected it is possible to cover $p_{1}(A \cap(P-T))$ by closed sets $B_{1}, \cdots, B_{k}$ that are pairwise disjoint and, for each $i=1, \cdots, k$, there is an $x_{i}$ such that $B_{i}$ lies in the $\epsilon_{x_{i}}-$ neighborhood of $x_{i}$. Define $f(x, y)=\left(x, y+t_{x_{i}}-t\right)$ for $x \in B_{i}$. Then extend $p_{2} \cdot f: \cup B_{i} \times E^{k} \rightarrow E^{k}$ to a map $f_{2}: B \times E^{k} \rightarrow E^{k}$ such that $d\left(f_{2}, p_{2}\right)$ $<\epsilon$. Then extend $f$ to $B \times E^{k}$ by setting $f=\operatorname{Id} \times f_{2}: B \times E^{k} \rightarrow B \times E^{k}$. Then $f(A) \cap P \subset T$. Now $f$ can be extended to an $\epsilon$-map of $E^{n}$ such that 
$p_{1} \cdot f=p_{1}: E^{n} \rightarrow E^{n-k}$. Thus $f \cdot h(\sigma) \cap P \subset T$. Since $P$ is locally tame at each point of $T$ there is an approximation $g$ of $f \cdot h$ such that $g(\sigma) \cap P$ $=\varnothing$. Thus $E^{n}-P$ is $1-\mathrm{ULC}$ and $P$ is tame in $E^{n}$.

Corollary 5.1. Let $C \subset E^{n}$ be an $m$-cell that factors 1-time. Let $P$ be a k-dimensional polyhedron topologically embedded in $C, k<m \leqq n-2$, and $n \geqq 5$. Then for each $\epsilon>0$ there is an $\epsilon$-push $H$ of $(C, P)$ such that $H(P)$ is tame in $E^{n}$.

Proof. This is actually a corollary to the proofs of Theorem 3 and Theorem 5. Let $K$ be a triangulation of $E^{n}$ and suppose $C=B \times I$ $C E^{n-1} \times E^{1}$. Then there is an approximation $j$ of the inclusion map $i$ : $\left|K^{2}\right| \rightarrow E^{n}$ such that $j\left(\left|K^{2}\right|\right) \cap C$ is a 0 -dimensional subset of $B \times\left\{t_{1}\right.$, $\left.\cdots, t_{p}\right\}$ for some numbers $t_{1}, \cdots, t_{p} \in I$. Thus for any $k$-dimensional polyhedron $P \subset C$, there is a small homeomorphism $h$ of $C$ such that $h(P) \cap j\left|K^{2}\right|=\varnothing$. Thus we can obtain by a sequence of such steps a small homeomorphism $H$ of $C$ such that $E^{n}-H(P)$ is 1-ULC. Thus $H(P)$ is tame.

REMARKs. Do Theorem 3 and Theorem 5 remain true if the hypothesis $n \geqq 5$ is replaced by $n=4$ ? Does Theorem 5 remain true if the hypothesis $m \leqq n-2$ is replaced by $m=n-1$ ? More specifically take Bing's 2-sphere $S \subset E^{3}[2]$. Are all subarcs of $S \times I \subset E^{4}$ tame?

Theorem 5 is sharp in the sense that there are examples of cells that factor $k$-times and for which some $(k+1)$-dimensional subcell is wild.

Daverman has independently proved Theorem 3 for the case $m=2$.

\section{REFERENCES}

1. R. H. Bing, Each disk in $E^{3}$ contains a tame arc, Amer. J. Math. 84 (1962), 583-590. MR 26 \#4331.

2. - $A$ wild surface each of whose arcs is tame, Duke Math. J. 28 (1961), 1-15. MR 23 \#A630.

3. R. H. Bing and J. M. Kister, Taming complexes in hyperplanes, Duke Math. J. 31 (1964), 491-511. MR 29 \#1626.

4. J. L. Bryant and C. L. Seebeck III, Locally nice embeddings of polyhedra, Quart. J. Math. Oxford Ser. (2) 19 (1968), 257-274. MR 38 \#2751.

5. - Locally nice embeddings in codimension three, Quart. J. Math. Oxford Ser. (2) 21 (1970), 265-272.

6. A. V. CernavskiY, Topological embeddings of manifolds, Dokl. Akad. Nauk SSSR 187 (1969), 1247-1250=Soviet Math. Dokl. 10 (1969), 1037-1041.

7. H. Gluck, Embeddings in the trivial range, Ann. of Math (2) 81 (1965), 195-210. MR 30 \#3456.

8. T. Homma, On the embedding of polyhedra in manifolds, Yokohama Math. J. 10 (1962), 5-10. MR 27 \#4236.

9. R. Miller, Approximating codimension 3 embeddings (to appear).

10. T. B. Rushing, Everywhere wild cells and spheres (to appear).

Michigan State University, East Lansing, Michigan 48823 